\title{
A microcomputer-controlled complex reaction time instrument
}

\author{
R. R. MARCUS, S. M. HORVATH, and J. A. GLINER \\ Institute of Environmental Stress, University of California, Santa Barbara, California 93106
}

\begin{abstract}
An inexpensive microcomputer system that measures complex reaction time to a visual stimulus is described. This system can determine the rate of gain of information by utilizing a two-, four-, or eight-choice reaction time task encompassed within the same unit. The system turns on randomly chosen lights and monitors the time required for the subject to depress a corresponding switch. Information describing the stimulus and the subject's response are printed on a Teletype after each response.
\end{abstract}

The reaction time system described is comprised of a microcomputer, a stimulus light panel, a response switchboard, a Teletype (TTY), and software (Figure 1). Its versatile design provides opportunity for developing other psychophysiological test systems in addition to that being described. These can be readily accomplished by modification of the software or construction of other peripherals. The materials required to build the microcomputer, stimulus light panel, and response switchboard cost approximately $\$ 1,000$.

\section{MICROCOMPUTER}

The microcomputer is constructed around a Motorola 6800 design evaluation card. This card contains a M6800 microprocessor, two peripheral interface adaptors (PIA), a current loop TTY interface,

This work was supported in part by the National Institutes of Health, under Grant NIH ESO1143. the MIKBUG system on a read-only memory (ROM), and a 128-word random-access memory (RAM) used by the MIKBUG system. We have interfaced $4 \mathrm{~K}$ words of RAM, a stimulus light panel, a response switchboard, and a real-time clock to this card. The 4K RAM and the interface circuitry for these other devices each reside on separate circuit boards in the microcomputer. The switches, lights, and real-time clock are interfaced to one PIA. The real-time clock produces interrupts at a rate of $1 \mathrm{kHz}$. The TTY is interfaced to the other PIA.

The M6800 microprocessor is a bidirectional, busoriented, 8-bit parallel machine with 16 bits of address and a minimum instruction time of 2 microsec. The MIKBUG system is used to load paper tapes into memory, print the contents of memory on the TTY, change the contents of individual memory locations, print the contents of computer registers, and start user programs. It provides a TTY handler and an interrupt handler that can be accessed by user programs to simplify input and output.

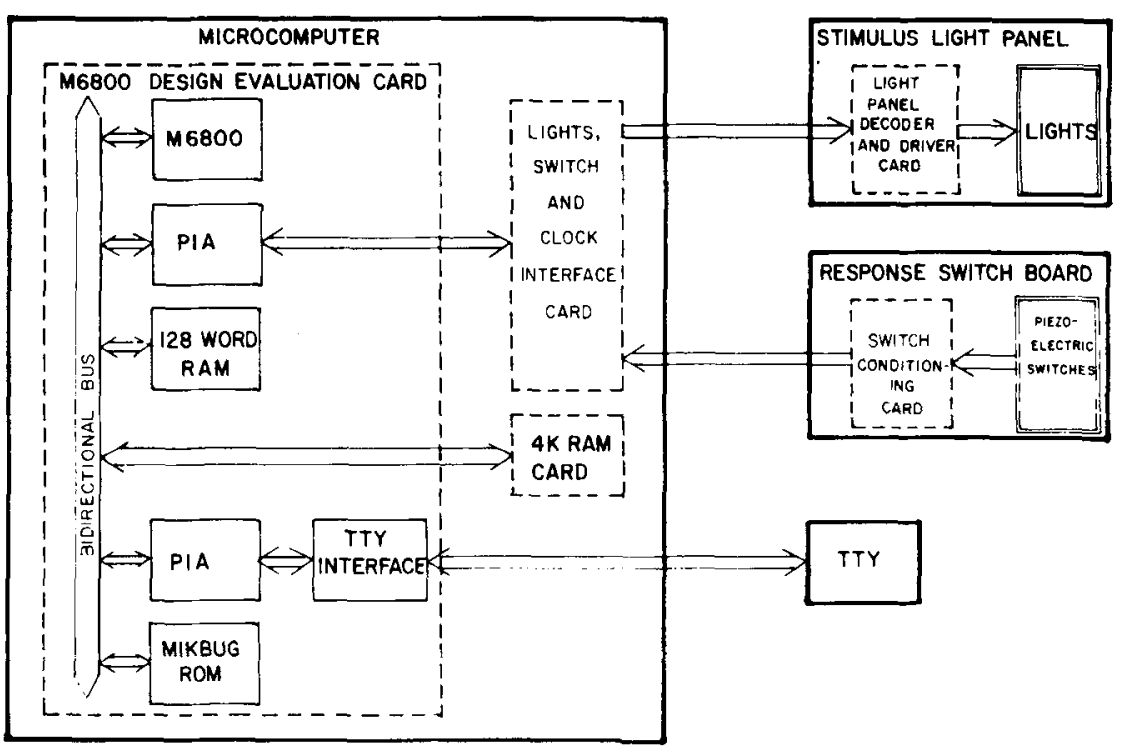

Figure 1. Block diagram of complete system. 
A marker output is provided for a recorder. In the present configuration, this output connects to the Grass polygraph driver amplifier inputs $\mathrm{J} 1$ and $\mathrm{J} 2$. It outputs a $1.0-\mathrm{sec}+.7 . \mathrm{V}$ level during the time when the stimulus is occurring. It outputs a $1.0-\mathrm{sec}-.7-\mathrm{V}$ pulse when an incorrect response is detected. This information, along with EEG, can be recorded on an instrumentation tape recorder by attaching the Grass driver amplifier outputs to the tape recorder inputs. The tape can later be played back to the PDP-12 computer for evoked-potential analysis.

\section{STIMULUS LIGHT PANEL}

A two-, four-, or eight-choice discrimination reaction time task is possible with the system in its present configuration. All three tasks, corresponding to 3,2 , and 1 bits of information, are visually displayed on a light panel (Figure 2). The three lights on the right side of the panel indicate to the subject the row to which he must respond.

The light panel contains 17 neon lamps behind red lenses. Neon lights were used because they come on almost instantly. Each light is driven with a relay that resides behind the light panel along with decoding circuitry. The red lenses are $15 \mathrm{~mm}$ in diam and are spaced on $25-\mathrm{mm}$ centers.

\section{RESPONSE SWITCHBOARD}

In a reaction time system, it is desirable to sense the subject's response with minimum delay added by the response sensor. Ordinarily, this is accomplished by position-sensing devices such as push-button switches or telegraph keys. However, with these devices, low operating force is required for minimum response time, and this often leads to inadvertent responses when used for extended periods of time. To avoid this weakness, a motion-sensitive sensor is more appropriate than a position-sensitive sensor. A capacitively coupled piezoelectric switch element was developed to satisfy this requirement.

A switchboard containing eight switch elements (Figure 3) spaced to provide the subject a comfortable

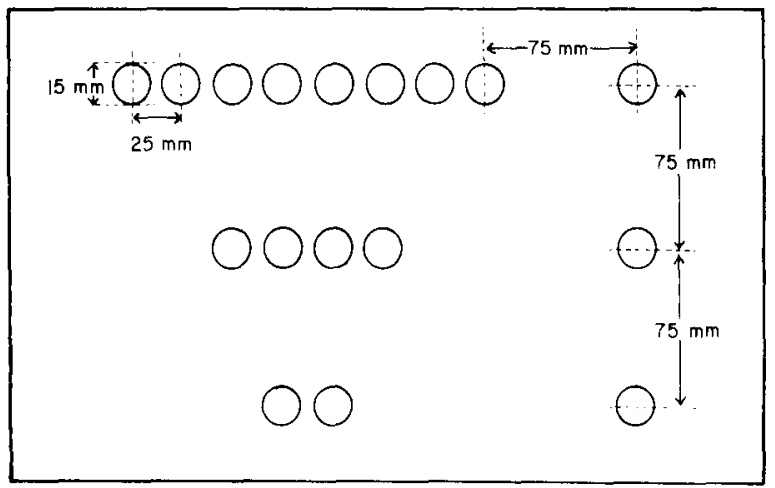

Figure 2. Spatial arrangement of lights on the panel.
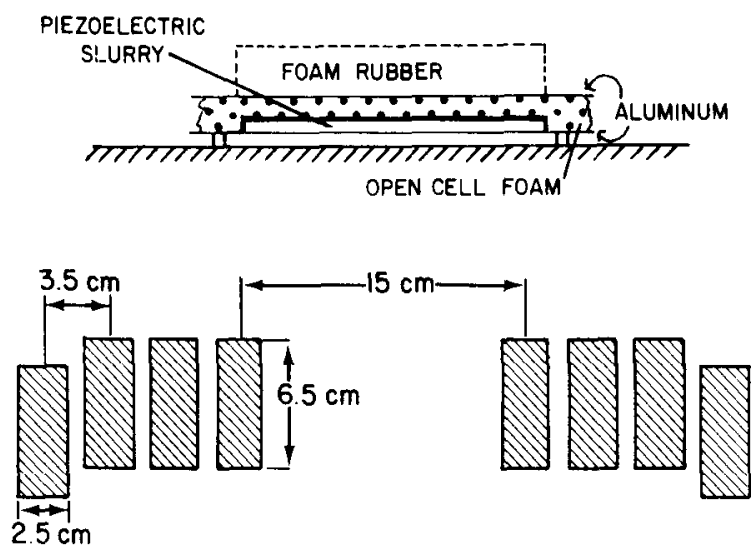

Figure 3. The upper section presents a cutaway diagram of the response switch. The lower section diagrams the position of the switches on the switch board.

place to rest his eight fingers (thumbs excluded) was constructed. Large switch elements were used to provide for a variety of hand sizes.

The switches are made of a sandwich of two pieces of aluminum with a piezoelectric crystal "painted" on the inside of one aluminum piece, as shown in Figure 3. This assembly is supported at both ends so that it bends when depressed on the foam rubber. Foam rubber provides a surface that moves when depressed, thus providing the subject with the "feel" of a switch.

The voltage produced by the piezoelectric crystal is proportional to the magnitude of the distortion received. The crystal is capacitively coupled to a gain-10 amplifier, A1 (Figure 4). The output of A1 is conditioned by a low-pass filter and compared to a potentiometer-selected voltage (sensitivity adjustment) by amplifier $\mathrm{A} 2$. When the voltage at $\mathrm{X}$ becomes less than the voltage at $\mathrm{Y}, \mathrm{A} 2$ outputs a positive level. A2 has some positive feedback to provide hysteresis, which prevents a noisy signal at X from looking like many switch closures. The output of $\mathrm{A} 2$ is conditioned to be TTL compatible with a resistor and a Zener diode. This circuit outputs a positive pulse each time the switch is depressed. An LM348 operational amplifier was used for $\mathrm{A} 1$ and $\mathrm{A} 2$.

\section{SOFTWARE}

The software for this system is a set of assembly language routines written for the M6800 microprocessor. By writing to and reading from the devices interfaced to the microprocessor, the software communicates with the test operator to set up an experimental run, generate the stimuli, monitor the subject response, and communicate the results to the test operator.

In order to facilitate software modification and maintenance, the programs are structured as a main routine and a set of subroutines. The main routine is entered from MIKBUG, the M6800 firmware utility package, and controls the experimental sequence from 


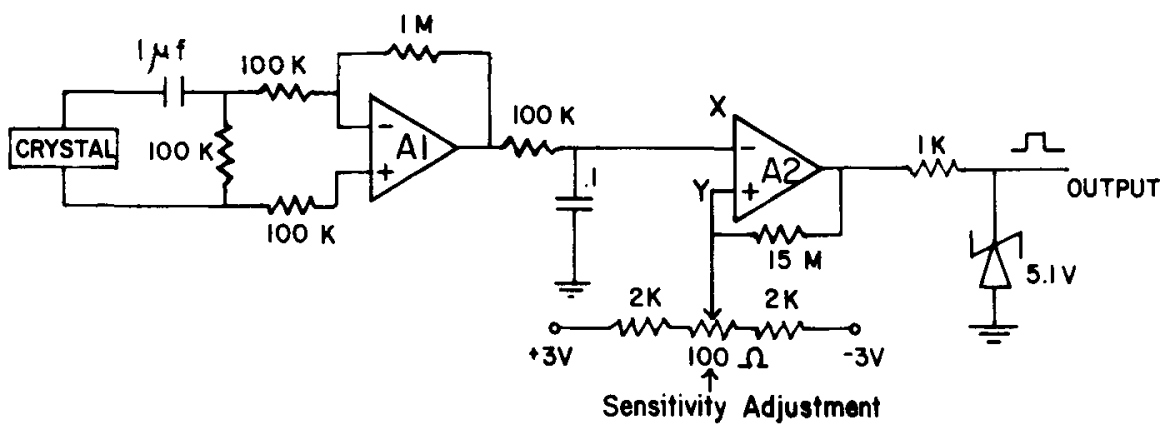

Figure 4. Schematic of response switch signal conditioner.

that point. It calls subroutines to perform the following functions: (1) Get initialization values from test operator for number of unique stimuli to use, delay time interval between consecutive stimuli, and variable starting point in the random number sequence that specifies stimulus order. (2) Initialize the input-output (I/O) devices and the internal clock. (3) Light the field indicator light in order to inform the subject of the light field in which the stimulus will appear. (4) Generate the next digit in the pseudorandom number sequence used to specify which light will be lit. (5) Delay a specified amount of time. (6) Light the selected stimulus light. (7) Monitor the subject's response. (8) Print the results of a stimulus-response trial on the TTY.

Some of these routines call other utility subroutines to perform the following functions: (1) Check the stimulus and response timer. (2) Convert a binary value to a string of decimal digits in a format to be printed on the TTY. (3) Suppress leading zeros on the values to be printed. (4) Generate a signal on the Grass recorder indicating that the subject's response to a stimulus light was not the correct one. In addition to these routines, there is a routine that is an interrupt nandler. It detects signals generated by the clock and updates the timer when they occur. It also detects the subject's switch response after a stimulus. The TTY I/O subroutines that are part of MIKBUG are also used by this package for its TTY communication.

The utility programs of the OS/8 operating system running on a Digital Equipment Corporation PDP-12 generate the machine language program that runs in the M6800. The OS/8 text editor is used to enter the assembly language routines described previously. A cross-assembler, designed to run on the PDP-12 and generate M6800 machine language from M6800 assembly language, is used to process each subroutine. OS/8 utilities are then used to punch the resulting machine language onto a paper tape in a format suitable to be read by the MIKBUG routine on the M6800. Since M6800 cross-assemblers are available for most leading minicomputers, the development of this system is not limited to PDP-12 users.
The modular structure of the programs and the capabilities of the PDP-12 make it fairly simple to modify a function of the system and generate a new machine language paper tape.

\section{OPERATION}

This system is easy to use and, once the tape is loaded, the operator need only respond to four computer questions. The loading procedure is started by depressing the reset button, which initializes the MIKBUG system. The TTY responds with an asterisk, which indicates that the system is properly loaded. The program tape is inserted into the TTY paper tape reader where it is loaded into memory by depressing " $L$ " on the TTY for each subroutine. When all the subroutines have been loaded, the MIKBUG system is used to load the program starting address.

The program is started by typing "G." The experimenter must respond to four questions about the configuration of the experiment. An example of this is:

1. ENTER 2, 4, OR 8 FOR THE NUMBER OF STIMULUS LIGHTS TO USE, ? 4

2. ENTER 2 HEX DIGITS TO INITIALIZE RAND1, ? 25

3. ENTER 2 HEX DIGITS TO INITIALIZE RAND2, ? SE

4. TIME BETWEEN STIMULI, ? 15

After the fourth question is answered, the TTY prints the heading (Table 1). The processor starts generating stimuli and recording the results on the TTY. The run number, an asterisk for incorrect responses, the response time, a number identifying the stimulus light, and a number identifying the response switch depressed are printed. Presentation of stimuli and recording of responses continue until the reset button is depressed. 
Table 1

Teletype Ouput While Running Reaction Time Processor Using Field 1

\begin{tabular}{ccccc}
$\begin{array}{c}\text { Run } \\
\text { Number }\end{array}$ & $\begin{array}{c}* \text { If } \\
\text { Wrong } \\
\text { Switch }\end{array}$ & $\begin{array}{c}\text { Delay } \\
\text { Time } \\
\text { (msec) }\end{array}$ & Light & Switch \\
\hline 1 & $*$ & 824 & 4 & 5 \\
2 & & 692 & 8 & 8 \\
3 & & 431 & 6 & 6 \\
4 & & 727 & 8 & 8 \\
5 & & 659 & 1 & 1 \\
6 & & 1250 & 3 & 3 \\
7 & & 508 & 1 & 1 \\
8 & & 961 & 4 & 4 \\
9 & $*$ & 587 & 5 & 8 \\
\hline
\end{tabular}

\section{CONCLUSION}

This compact system has been developed at a low cost (approximately $\$ 1,000$ for parts), is simple to use, and provides an output that is easy to analyze but is sufficiently complex to provide a task that will allow systematic investigation of channel capacity in man. It is being utilized in our laboratory to measure central nervous system changes to different environmental stressors.

(Received for publication April 14, 1978; revision accepted June 19,1978 .) 